\title{
A Study of Reactive Power Requirements of Traditional and Restructured Power System
}

\author{
Laleh Haddadi ${ }^{1, *}$, Abolfazl Pirayesh Neghab ${ }^{2}$, Seid Babak Mozafari ${ }^{1}$, \\ Morteza Ghasem Salamroodi ${ }^{3}$ \\ ${ }^{1}$ Department of Power Engineering, Science and Research Branch, Islamic Azad University of Tehran, Tehran, Iran \\ ${ }^{2}$ Department of Power Engineering, Shahid Beheshti University, Tehran, Iran \\ ${ }^{4}$ Power Distribution Company of West of Mazandaran, Noshahr, Iran
}

Email address:

1_haddadi90@yahoo.com (L. Haddadi), ab_pirayesh@yahoo.com (A. P. Neghab), mozafari_babak@yahoo.com (S. B. Mozafari), msalamroudi@yahoo.com (M. G. Salamroodi)

\section{To cite this article:}

Laleh Haddadi, Abolfazl Pirayesh Neghab, Seid Babak Mozafari, Morteza Ghasem Salamroodi. A Study of Reactive Power Requirements of Traditional and Restructured Power System. Journal of Electrical and Electronic Engineering. Special Issue: Research and Practices in Electrical and Electronic Engineering in Developing Countries. Vol. 3, No. 2-1, 2015, pp. 101-106. doi: 10.11648/j.jeee.s.2015030201.31

\begin{abstract}
Due to importance of requirements of reactive power in a power system, by employing probabilistic load flow with Monte Carlo method we can gain the exact value of requirement reactive power of system in a traditional and a restructured power system. In this paper the main object is the examination of effect of random changes in load, generation and price on all requirement reactive power of system as well as the amount of reactive power of each bus. Effective parameters in reactive power distribution are determined and an effective probable model for each of parameters is presented. The simulation results are used by network management for reactive power and voltage control service contracts. The results of analysis in two environments (traditional and restructured power system) are shown. The Monte Carlo method and MATLAB software is used, and studies are done on 30 buses IEEE system that is modeled by MATPOWER software.
\end{abstract}

Keywords: Reactive Power, Restructured Power System, Monte Carlo Simulation

\section{Introduction}

Reactive power supply is essential for reliably operating the electric transmission system. Inadequate reactive power has led to voltage collapses and has been a major cause of several recent major power outages worldwide.

The rules for procuring reactive power can affect whether adequate reactive power supply is available, as well as whether the supply is procured efficiently from the most reliable and lowest- cost sources.

Not only is reactive power necessary to operate the transmission system reliably, but it can also substantially improve the efficiency with which real power is delivered to customers. Increasing reactive power production at certain locations (usually near a load center) can sometimes alleviate transmission constraints and allow cheaper real power to be delivered into a load pocket [1]

In the other hand Estimation of reactive power more than requirement value will cause additional costs and will occupy capacity of reactive power sources that have other capabilities, like generators. Evaluation of requirements of reactive power for system is very important.

In this paper by using probabilistic load flow with Monte Carlo method we gain the exact value of requirement reactive power of system in a traditional and a restructured power system.

The remaining section of the paper is organized as follows: In section 2 we will review restructured power system, In section 3 we examine the pool market, probabilistic and optimal load flow, probabilistic load flow using Monte Carlo method in section 4 and the results of the case study will be described in section 5 and 6 .

\section{Restructured Power System}

Power industry are changing and moving in a direction that by allowing producers to competition and market conditions, try to reduce the costs of producing and distributing electricity, eliminate certain inefficiencies, increase customer choice. This evolution towards a competitive electricity market often 
called deregulation or restructuring and includes below benefits:

1 Providing choice for consumers

2 Creation suitable condition for providing better service

3 Competitive supply of electrical products at different levels and consequently set reasonable prices for consumers

4 Attract investments in the private sector and guidance for public benefit no need for massive public investment

5 Offered products quality enhancement with respect to the competition and...

\section{Pool Market Model, Probabilistic and Optimal Load Flow}

In the pool market model, there are two main sides of entities participating in the market, i.e. customer and supplier. The pool operator takes electricity transaction bids and offers from these two entities and dispatches them in an economic manner depending on the price and MW biddings. In general, the customers and suppliers do not directly interact to each other, but only indirectly through the pool operator. [2] In the pool market, participants post selling offers for a certain amount of electrical energy at a specified price and in a period of time. These offers are sorted by price ascending. And produce market supply curve. And also, the market demand curve is obtained from amount and price of customer requests which are arranged descending. Intersection of supply and demand curves is represented market equilibrium.

In order to implement the restructured power system in this paper we use pool market model.

Probabilistic load flow is a macroscopic random method under steady-state operating conditions of the power system, which considers different random factors on the performance of power system.[3] To consider the uncertainty in the power system probabilistic load flow is a proper way that in it uncertainty in the system parameters are considered as random variables. In this paper probabilistic load flow using Monte Carlo simulation is done.

Optimal load flow is a nonlinear optimization to determine the optimum parameters in a power system that has an objective function and number of constraints. Here the objective function is total cost of production of active or reactive power or both. These costs may be patchy or linear functions of the output of the generator polynomial functions are defined. The formulation is as follows:

$$
\begin{gathered}
\min _{P g, Q g} \sum f_{1 i}\left(P_{g i}\right)+f_{21}\left(Q_{g i}\right) \\
P_{g i}-P_{L i}-P(V, \theta)=0 \\
Q_{g i}-Q_{L i}-Q(V, \theta)=0 \\
\widetilde{S_{l J}^{f}} \leq S_{i j}^{\max } \\
\widetilde{S_{l J}^{t}} \leq S_{i j}^{\max }
\end{gathered}
$$

$$
\begin{gathered}
V_{t}^{\min } \leq V_{t} \leq V_{t}^{\max } \\
P_{g i}^{\min } \leq P_{g i} \leq P_{g i}^{\max } \\
Q_{g i}^{\min } \leq Q_{g i} \leq Q_{g i}^{\max }
\end{gathered}
$$

In this paper, by using probabilistic optimal load flowproduction changes, load changes and active and reactive cost changes in a power system are considered. And also Monte Carlo simulation method is used to determine amount of reactive power demand in a pool market .

\section{Probabilistic Load Flow Using Monte Carlo Method}

Monte Carlo simulation is a process that occurrence of modes in all its aspects is purely coincidental. In Monte Carlo simulation, a random number generator is used to model the occurrence of events. This behavior is desirable, because by repeating the simulation, a probability distribution of random numbers will be obtained. And the mean, median, variance and statistical parameters will be calculated.[8] In this paper, by using probabilistic load flow and probabilistic optimal load flow production changes, load changes and active and reactive cost changes in a power system are considered. And also Monte Carlo simulation method is used to determine amount of reactive power demand in a power system.

To consider the uncertainty in the power system probabilistic load flow is a proper way that in it uncertainty in the system parameters are considered as random variables. In this paper, using Monte Carlo method, uncertainty of the system production and loads are modeled as random variables And with running the program and probabilistic load flow and probabilistic optimal load flow for about 8000 to 10000 times, probability distribution of parameters of system be obtained and the amount of requirements reactive power of the system in a traditional and restructured power system are obtained. MATLAB software was used to generate normal random numbers and the required random numbers are generated.

Probabilistic load flow using Monte Carlo method is the most comprehensive tool to evaluate different scenarios for uncertainty in a system. In Monte Carlo simulation at first the base network that in it the network structure is characterized by the production of generator and load bus is selected, then to the following, load flow is done:

Using a random normal distribution function a random amount of load for a load bus is selected.

The selected bus is modified with the new value selected

Using Newton-Raphson load flow network status can be evaluated and the amount of output parameters is characterized.

If the stopping criterion is provided by the simulation, the simulation ends, otherwise, items 1 to 3 are repeated. Stopping criterion can determine the number of iterations for Monte Carlo simulation or the specific coefficient of variation is.[9] 
With the finishing Monte Carlo simulation, average value of the output parameters obtained from the following equation can be used as the best estimation:

$$
\overline{\mathrm{A}}=\frac{1}{\mathrm{~N}} \sum_{i=1}^{\mathrm{N}} A_{i}
$$

And the variance of output parameters can be obtained from the following equation:

$$
\mathrm{V}=\frac{1}{\mathrm{~N}} \sum_{\mathrm{i}=1}^{\mathrm{N}}\left(\mathrm{V}_{\mathrm{k}}-\overline{\mathrm{V}}\right)^{2}
$$

Where $\bar{M}$ average output parameters, $M_{k}$ simulation output parameter at the $\mathrm{k}$ th iteration and $\mathrm{N}$ is the number of samples tested.

In this paper that the output parameter is the system reactive power, Formulas 1 and 2 are as follows:

$$
\begin{gathered}
\bar{Q}=\frac{1}{\mathrm{~N}} \sum_{\mathrm{i}=1}^{\mathrm{N}} \mathrm{Q}_{\mathrm{i}} \\
\mathrm{V}=\frac{1}{\mathrm{~N}} \sum_{\mathrm{i}=1}^{\mathrm{N}}\left(\mathrm{Q}_{\mathrm{i}}-\overline{\mathrm{Q}}\right)^{2}
\end{gathered}
$$

Where $\bar{Q}$ average output reactive power, $Q_{i}$ amount of reactive power at the $\mathrm{i}$ th iteration and $\mathrm{N}$ is the number of samples tested.

\section{Case Study}

The study is done on 30 buses IEEE system that has 6 generator buses and 24 load buses. It is shown in figure1:

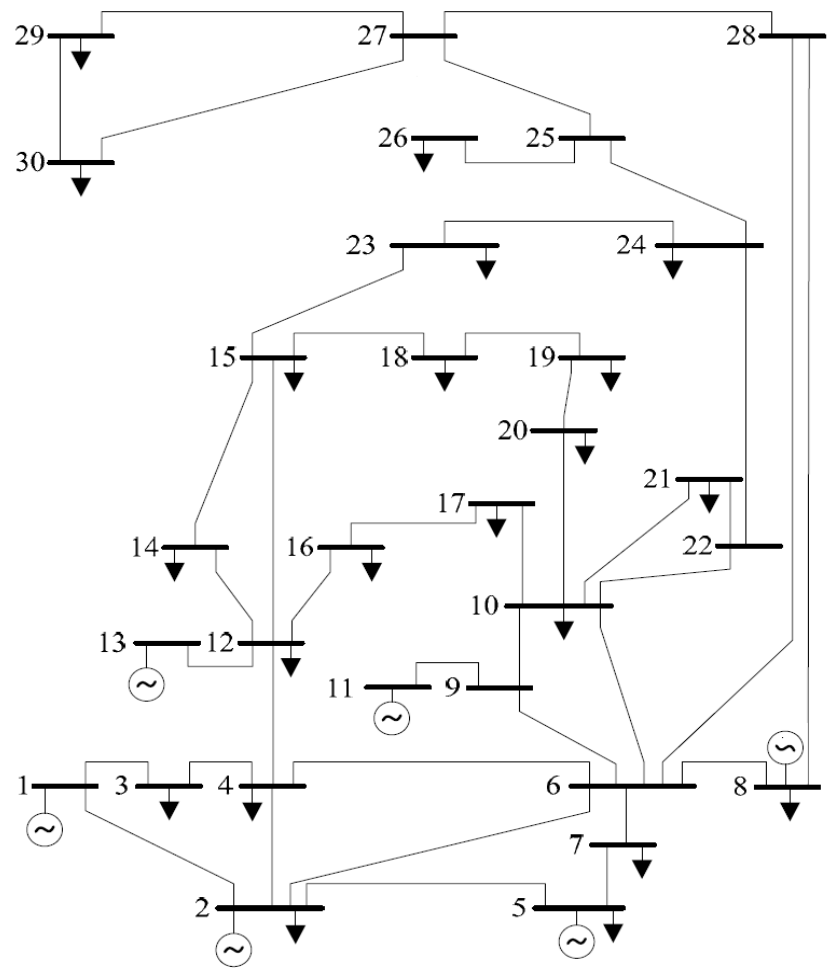

Figure 1. 30 buses network diagram

Algorithm based on Monte Carlo simulation is as follows: Count $=0$ generate uncertainty random numbers of production, load, or cost

Insert random numbers in the original system and modify system now

Do normal load flow or optimal load flow

Count +1 .

If converged, go to step 8 .

If did not converge, count-1, now and Go to Step 2

Do Monte Carlo simulation to saturate system parameters

If converged, go to the last step

If not converged, go to step 2

The flowchart of proposed method is illustrated in figure 2 .

Compute the average reactive power requirements of the network

To evaluate the system in a traditional system we used Newton Raphson load flow, with considering different probabilities in load and production and doing 10000 iterations for each mode, the amounts of the requirement reactive power at each bus and all system obtain.

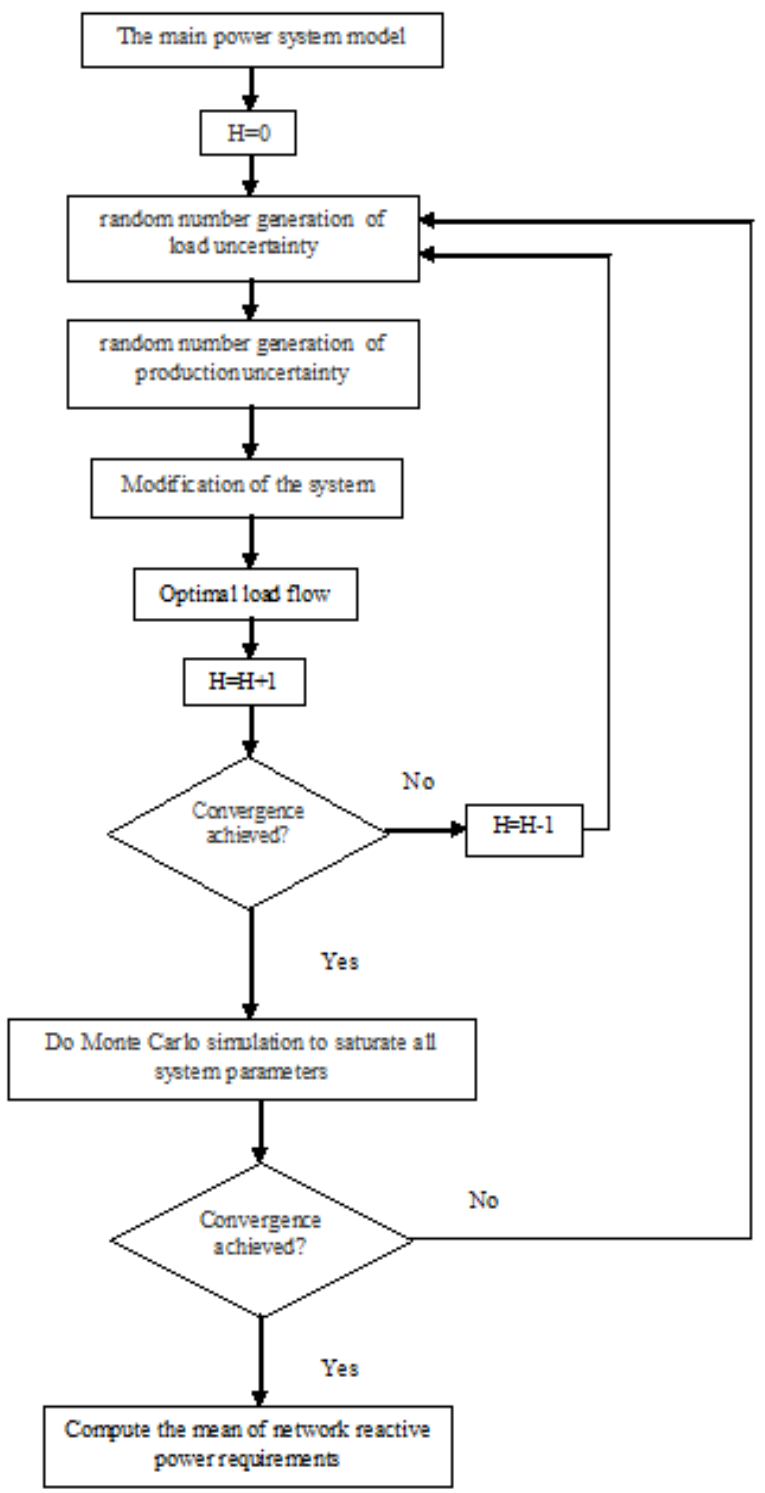

Figure 2. Flowchart of proposed method 
Loads of power system is variable and uncertain. Different probability distribution functions may be used to show uncertainty. In this paper we used normal distribution

Figures 3, shows Random variation of active load consuming at bus 2 . for generating normal random number," randn "rule in MATLAB software is used. For example in order to generate random numbers of active load consuming at bus 2 following expression is used:

$$
\mathrm{x}=21.7+7.23 \mathrm{randn}(1)
$$

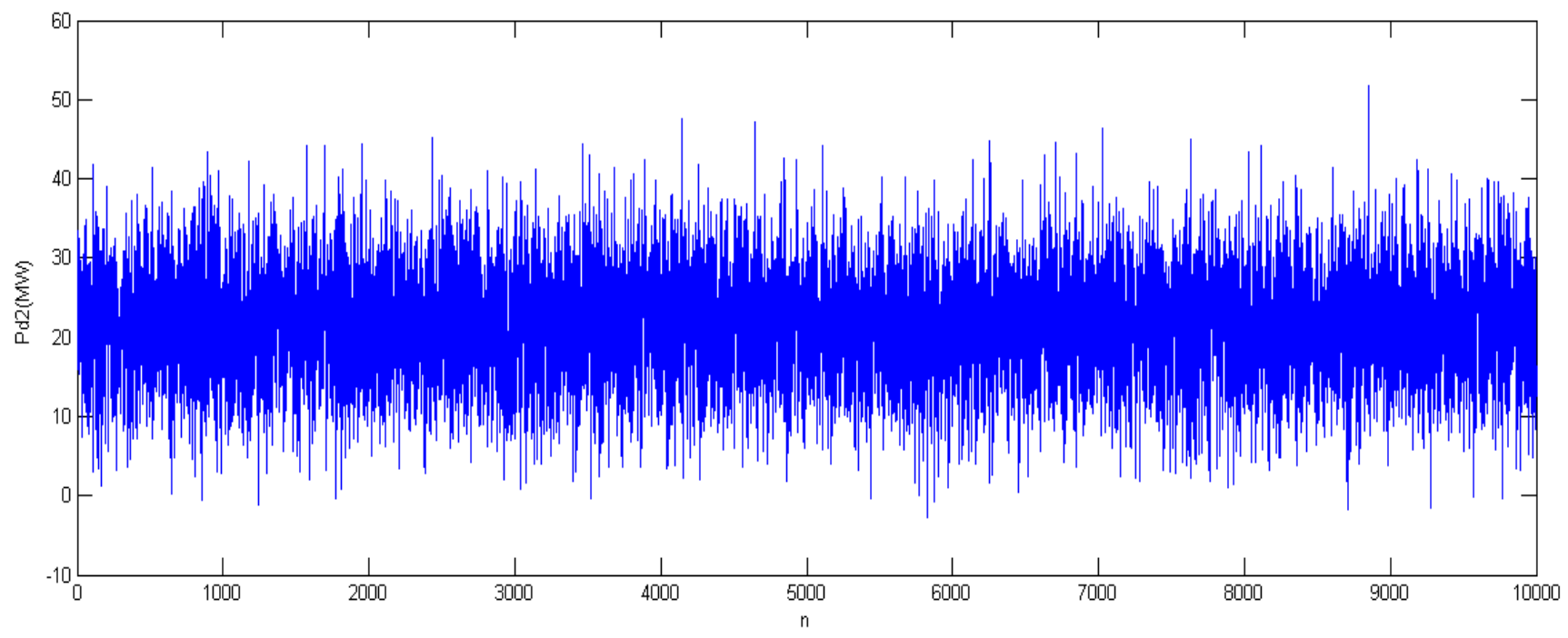

Figure 3. Diagram of random variation of active load consuming at bus 2

To evaluate the system in a restructured system and in a pool market, in the above algorithm, optimal load flow is used instead of the ordinary load flow. And moods of production, load and price changes are considered as a normal random distribution. This time the number of iterations of simulation is 8000 times.

Figures 4 and 5 are shown examples of the diagrams of mean changes of reactive power generation at bus 1, 2, 5, 8, 11, 13, and the whole system, result of random changes in the parameters. Results of a number of moods are shown in tables 1 and 2.

Table 1. The results of the required reactive power in a traditional power system

\begin{tabular}{|c|c|c|c|c|}
\hline $\begin{array}{ll} & \text { Average of Reactive power random changes } \\
\text { Random States } & \\
\end{array}$ & Bus5 & Bus8 & Bus13 & $\begin{array}{l}\text { Total of } \\
\text { System }\end{array}$ \\
\hline Change of all loads & 39.3955 & 36.5619 & 10.5669 & 136.6483 \\
\hline Heavy loading & 39.9907 & 39.8906 & 11.1937 & 147.0116 \\
\hline Result of Monte Carlo simulation & 35.9175 & 36.3037 & 10.4899 & 134.7411 \\
\hline Optimal load flow with a medium value & 35.66 & 36.11 & 10.45 & 133.93 \\
\hline
\end{tabular}
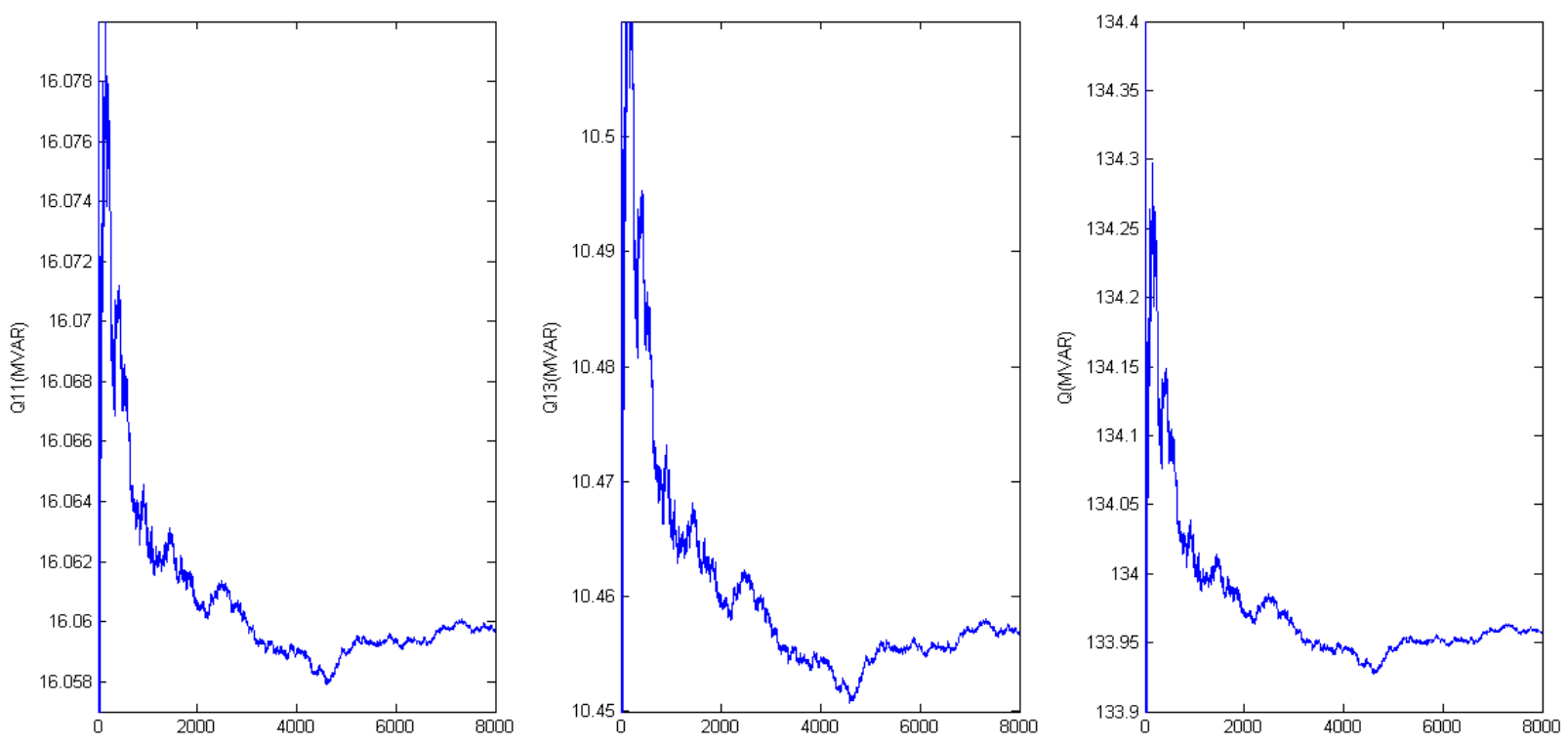

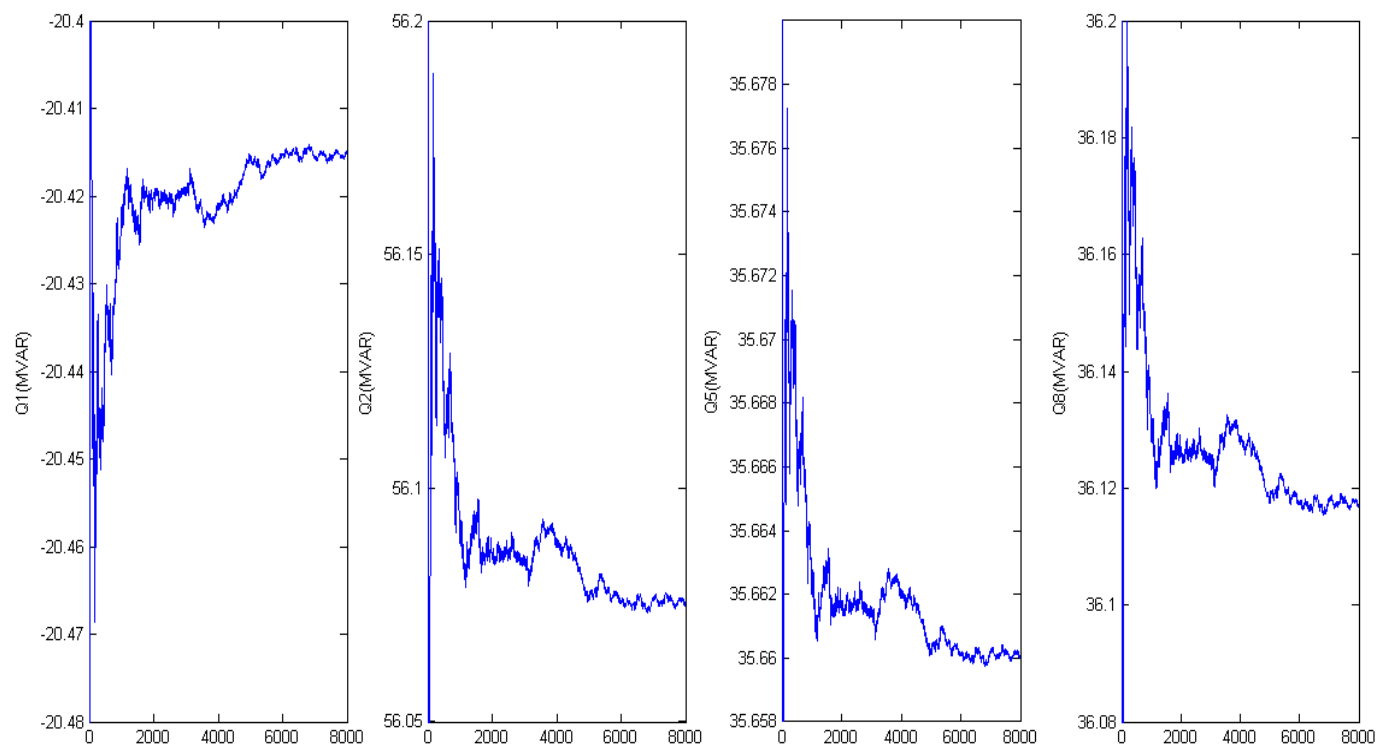

Figure 4. Diagram of the system reactive power changes in a traditional power system due to random variations in load at bus 15
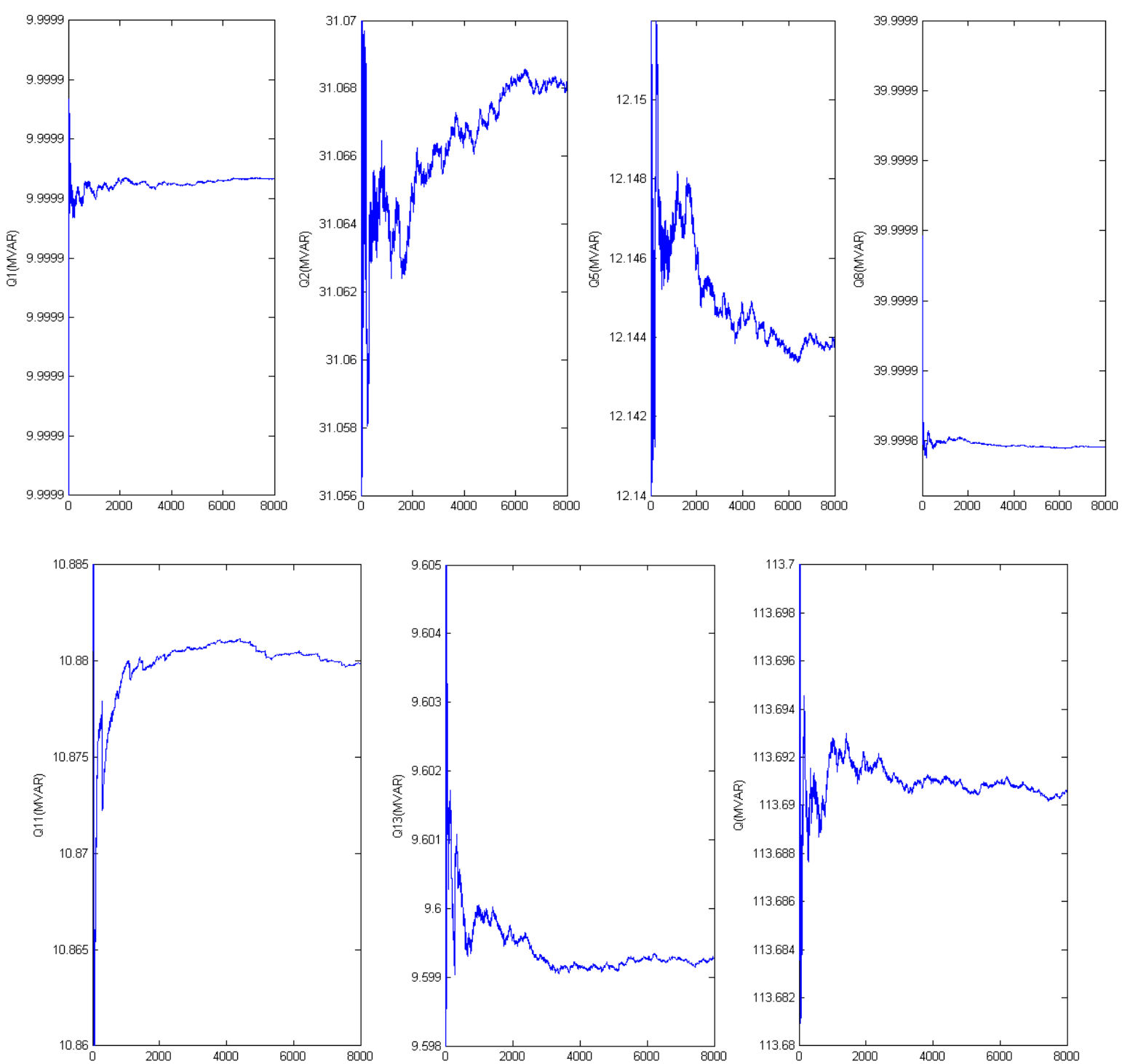

Figure 5. Diagram of the system reactive power changes in a pool market power system due to random variations in active power cost at bus 11 
Table 2. The results of the required reactive power in a pool market

\begin{tabular}{|c|c|c|c|c|}
\hline Average of Reactive power random changes & Bus5 & Bus8 & Bus13 & $\begin{array}{l}\text { Total of } \\
\text { System }\end{array}$ \\
\hline Change of all loads & 12.137 & 39.9999 & 9.5826 & 113.8114 \\
\hline Heavy loading & 31.4943 & 40.2744 & 11.8362 & 146.2001 \\
\hline Optimal load flow with a medium value & 12.14 & 40 & 9.6 & 113.69 \\
\hline
\end{tabular}

Cost function is a function of polynomial system (14) considered. Where the coefficients are shown in Appendix Tables 3 and 4 .

$$
\mathrm{f}(\mathrm{p})=C_{3} P^{3}+C_{2} P^{2}+C_{1} \mathrm{p}+C_{0}
$$

Table 3. Active power generation cost coefficients

\begin{tabular}{llllll}
\hline Bus & Startup & Shutdown & \multicolumn{1}{c}{$\boldsymbol{C}_{\mathbf{2}}$} & $\boldsymbol{C}_{\boldsymbol{1}}$ & $\boldsymbol{C}_{\mathbf{0}}$ \\
\hline 1 & 0 & 0 & 0.038432 & 20 & 0 \\
2 & 0 & 0 & 0.25 & 20 & 0 \\
5 & 0 & 0 & 0.01 & 40 & 0 \\
8 & 0 & 0 & 0.01 & 40 & 0 \\
11 & 0 & 0 & 0.01 & 40 & 0 \\
13 & 0 & 0 & 0.01 & 40 & 0 \\
\hline
\end{tabular}

Table 4. Reactive power generation cost coefficients

\begin{tabular}{llllll}
\hline Bus & Startup & Shutdown & $\mathbf{C}_{2}$ & $\mathbf{C}_{\mathbf{1}}$ & $\mathbf{C}_{\mathbf{0}}$ \\
\hline 1 & 0 & 0 & 0.02 & 0 & 0 \\
2 & 0 & 0 & 0.0175 & 0 & 0 \\
5 & 0 & 0 & 0.0625 & 0 & 0 \\
8 & 0 & 0 & 0.00834 & 0 & 0 \\
11 & 0 & 0 & 0.025 & 0 & 0 \\
13 & 0 & 0 & 0.025 & 0 & 0 \\
\hline
\end{tabular}

\section{Discussion and Conclusion}

In this paper by using Monte Carlo simulation, that is a flexible method, we found the exact amount of reactive power is needed for a restructured and a traditional power system. To do this we used the method of probabilistic optimal load flow and considered the uncertainties of production, load and cost and obtained the results of reactive power changes of different buses and also all buses that is the amount of total requirement of reactive power for system. One of states of uncertainty considered is a variable heavy loading of system. That is shown the maximum need of reactive power in a critical condition and without collapse.

Simulation is down on 30 buses IEEE system. The results are shown in section 5. Result of comparison between restructured and traditional is shown in tables 1 and 2.

\section{References}

[1] "Principles for Efficient and Reliable Reactive Power Supply and Consumption" STAFF REPORT Docket No. AD05-1-000 February 4, 2005

[2] Surachai Chaitusaney, and Bundhit Eua-Arporn, "Actual Social Welfare Maximization in Pool Market” IEEE 2002.

[3] X.Wang , J.R.McDonald "Modern power system planning" IEEE 2002

[4] Majid Oloomi Buygi, Gerd Balzer, Hasan Modir Shanechi, Mohammad Shahidehpour,"Market-Based Transmission Expansion Planning" IEEE Transaction On Power Systems, VOL. 19, NO. 4 Nov.2004

[5] Walid El-Khattam, Y.G.Hegazy, M.M.A.Salama“'Investigating Distributed Generation Systems Performance Using Monte Carlo Simulation” IEEE 2006.

[6] Roy Billinton- Ronald Allen"Reliability Evaluation of Engineering Systems" Plenum Press 1994.

[7] Richard E. Brown "Electric Power Distribution Reliability" Marcel Dekker, Inc. 2002.

[8] Mehrdad Hojjat, Habib Rajabi Mashhadi Optimal Dispatch of Power System Restructuring in considering the impact of the independent random variablesranian conference on electrical engineering, Tabiat Modares University 2008.

[9] Hasan Abniki, Hasan Monsef, Mina Jafari Inanlo "Flash estimate voltage using a Monte Carlo method" International Power System Conference, Tehran 2011.

[10] Maryam Ramezani,Mohammad Reza Khalghani, Hamid Falaghi "Probabilistic load flow in the power systems, wind power plants based on classified data" International Power System Conference, Tehran 2011.

[11] Laleh Haddadi, Abolfazl Pirayesh Neghab, Seid Babak Mozafari "Analysis of requirement reactive power for a restructured power system using Monte Carlo method" Journal of Basic and Applied Scientific Research, ISSN: 2090-4304, sep. 2013 